\title{
Predicting of the Residual Resource of Car Assemblies
}

\author{
Ivanov Vladimir, Vigerina Tatyana, Pilipenko Stanislav \\ Department of Road Transport, State University of Polotzk, Novopolotsk, Republic of Belarus \\ Email address: \\ ivprem@tut.by (I. Vladimir), t.vigerina@psu.by (V. Tatyana), s.pilipenko@psu.by (P. Stanislav)
}

\section{To cite this article:}

Ivanov Vladimir, Vigerina Tatyana, Pilipenko Stanislav. Predicting of the Residual Resource of Car Assemblies. International Journal of Transportation Engineering and Technology. Vol. 5, No. 4, 2019, pp. 68-73. doi: 10.11648/j.ijtet.20190504.11

Received: February 13, 2019; Accepted: October 12, 2019; Published: October 26, 2019

\begin{abstract}
The research paper introduces a method that can be used for the forecasting the residual life of automobile aggregates (through the example of automobile engines). The results of a test-drive have shown that the proposed method is less labor intensive and has a satisfactory forecast accuracy. In the research the tenets of the reliability theory and mathematical statistics were used as well as information on the post-repair operating time of repaired engines based on the value of the initial main parameter (the gap between the piston and cylinder) for 41 engines. The probability density of this parameter follows the Gauss' law. In our work we accept the nonlinear change in the mathematical expectation of the main parameter depending on the operating time in the form of a power law. The probability density of the aggregate resource is distributed according to the Weibull law. Adequacy of theoretical information to experimental data was determined by the Fisher criterion. The forecasting of the residual life of the aggregates is relevant when the operating time approaches their limit state. The relative forecast error varies from 0.021 to 0.130 , which is quite acceptable for the real-world applications.
\end{abstract}

Keywords: Assembly, Reliability, Longevity, Diagnostic Parameter, Residual Resource, Prediction

\section{Introduction}

Information about the remaining resource of car assemblies is necessary for planning the data and volumes of repair with determination of the demand for replacement. There are various methods for residual aggregate life [1-9]. The most simple, though approximate method for determination of remaining resource of a car assembly is based on the linear program building, when the change of the main parameter depending on the operating age is assumed as linear. In this case the remaining resource of a car assembly $t_{\text {rem }}$ is determined by the formula [10]:

$$
t_{\text {rem }}=t_{c u r}\left(\frac{P_{\text {lim }}-P_{\text {in }}}{P_{c u r}-P_{i n}}-1\right)
$$

here $t_{c u r}$ is the current operating age of a car assembly from the beginning of exploitation (operation) or after repair; $P_{\text {in }}$ and $P_{l i m}$ are the initial and limiting value of the main parameter correspondingly; $P_{c u r}$ is the current value of the main parameter in the moment of determination of the technical state. (See [1]).

The car assembly is put into operation after manufacture or overhaul with initial value of the main parameter $P_{\text {in }}$ (which is called in technical literature a structural parameter). The change of parameter during the running in is usually neglected because of its infinitesimal. The limiting value of the parameter $\mathrm{P}_{\text {lim }}$ conforms to the technical state of the assembly when it is not able performing the specified function because of wearing or deteriorating with time or performing of this function is connected with big consumption of resources. In majority of cases we have $P_{\text {in }}<P_{\text {cur }}<P_{\text {lim }}$.

The change of the main parameter depending on the operating age $t_{c u r}$ is often described by the power equation.

$$
P_{\text {cur }}=P_{\text {in }}+k t_{\text {cur }}^{\alpha},
$$

where $k$ is the coefficient, which had been obtained on the base of experimental data; $\alpha$ is the index of power, which characterizes the speed of changing the main parameter.

At the linear change of parameter $\alpha=1$, the speed of the change of parameter $P_{\text {cur }}$ during the time of the assembly service is constant. At $\alpha>1$ the value of parameter $P_{\text {cur }}$ is continuously increasing, and the curve of deviations of $P_{c u r}$ is concave. Actually the value of $\alpha$ for different assemblies is fluctuating in the limits 1.1-1.8 and more [10]. According to the data of investigations, the values of $\alpha$ while predicting the 
remaining resource ( for example, for motor and tractor engines), are assumed, with sufficient for practical purposes accuracy, equal to [11] (Table 1):

Table 1. The values of $\alpha$ for various parameters of the engines.

\begin{tabular}{ll}
\hline The harameters & The values \\
\hline By the break of gases into carter & $1.3-1.5$ \\
By the waste of carter oil & 2.0 \\
By capacity of engine & $1.1-1.7$ \\
By clearances in bearings of crankshaft & $1.4-1.6$ (See [2]). \\
\hline
\end{tabular}

In this case at the linear change of the main parameter $P_{\text {cur }}$ the remaining resource of the assembly, depending on the operating age, is determined by the formula:

$$
t_{\text {rem }}=t_{\text {cur }}\left[\left(\frac{P_{\text {lim }}-P_{\text {in }}}{P_{\text {cur }}-P_{\text {in }}}\right)^{\frac{1}{\alpha}}-1\right]
$$

With purpose to increase the accuracy of predicting the remaining resource, it is recommended to use a few values of the main parameter. obtained by the results of the previous measurements. The general number $m$ of these values should be equal in this case to 3-5 (at $m=2$ the error of prediction decreases unimportantly, at $m>5$ the rise of accuracy isn't practically observed).

Assuming that distribution of remaining resource of an assembly is submitted to Weibull's distribution, the remaining resource $t_{\text {rem }}$ (with the given probability of faultless operation) is [12]:

$$
t_{\mathrm{rem}}=t_{\mathrm{cur}}\left[\left(\frac{P_{\mathrm{lim}} / P_{\text {cur }}+5 \sigma_{\mathrm{p}}}{1+Б \sigma_{\mathrm{p}}}\right)^{1 / \alpha}-1\right]
$$

here $E$ is the tabulated integral of probabilities (quantil); $\sigma_{p}$ is the root-mean-square of the prediction error. When the quantil is equal to 0 , formula is transformed into the formula of remaining resource of an element at the smooth realizations of its parameter.

The considered methods for determination of remaining resource of the car assemblies are labor-consuming and do not ensure the necessary accuracy of prediction of latter.

\section{The Aim of the Work}

Increasing accuracy of prediction of remaining longevity of car assemblies with decrease of labor-input in determination of this quality.

\section{Methods of Investigation}

Authors used in their work the statements of the theory of reliability and mathematical statistics as well as information about after-repairing operating age of engines, which have been submitted to repair.

The labor-consuming industrial experiment had been carried out with purpose to study after-repairing operating age of 8-cylinder two-row petrol engines with working volume 4.251 depending on accuracy of the main geometric parameter (clearance between sleeve and piston), which characterizes to the greatest degree the technical state of an assembly. The contents of the experiment were following. The work had been carried out without interference into technological processes and organization of renewal of details and assembling of engines. Dimensions of details entering in joints had been measured by means of nutrometers and micrometers on the working places of their renewal, and subsequently values of parameter had been calculated in the form of locking dimension. Fields of parameter determination are limited on the left by minimum values fixed by the normative documents (specifications) for detail renewal, and on the right by the maximum values revealed in the course of their measurement. The cases when the values of parameters-arguments exceeded substantially normative ones (for example at the output of production conditioned by especial sanctions) were of the great interest for investigation. Deviations of detail parameters from normative values had been occasioned by using the spare parts of inferior quality, insufficient possibilities of metal-cutting equipment and using the details from repairing fund as their parameters according to the guide in capital repairs of assemblies are inferior to parameters of details, which have been manufactured at automobile works.

Details were arriving to the working places of their assemblage and were mounted on engines. A data card had been drawn up for each of these engines indicating the details with values of their dimensions. Workers of the laboratory of reliability established relations with organizations where these engines were operating. For each of these engines, information had been obtained about their operating age till the ultimate state (Table 2). Information had been obtained for 41 engines.

Table 2. Operating age t(thousands kms of run) of repaired engines till the ultimate state depending on the initial clearance $P_{\text {in }}(\mathrm{mm})$ between piston and cyl-

\begin{tabular}{|c|c|c|c|c|c|c|c|c|}
\hline № & $\boldsymbol{P}_{\text {in }}$ & $t$ & № & $\boldsymbol{P}_{\text {in }}$ & $t$ & № & $P_{\text {in }}$ & $T$ \\
\hline 1 & 0.07 & 91 & 15 & 0.09 & 104 & 29 & 0.09 & 105 \\
\hline 2 & 0.09 & 95 & 16 & 0.11 & 69 & 30 & 0.07 & 128 \\
\hline 3 & 0.10 & 121 & 17 & 0.11 & 78 & 31 & 0.08 & 130 \\
\hline 4 & 0.09 & 112 & 18 & 0.09 & 98 & 32 & 0.09 & 123 \\
\hline 5 & 0.11 & 70 & 19 & 0.06 & 120 & 33 & 0.12 & 59 \\
\hline 6 & 0.09 & 103 & 20 & 0.10 & 87 & 34 & 0.11 & 74 \\
\hline 7 & 0.12 & 55 & 21 & 0.08 & 119 & 35 & 0.10 & 87 \\
\hline 8 & 0.13 & 40 & 22 & 0.09 & 105 & 36 & 0.11 & 76 \\
\hline 9 & 0.10 & 89 & 23 & 0.07 & 144 & 37 & 0.09 & 105 \\
\hline
\end{tabular}
inder. 


\begin{tabular}{lllllllll}
\hline № & $\boldsymbol{P}_{\text {in }}$ & $\boldsymbol{t}$ & № & $\boldsymbol{P}_{\text {in }}$ & $\boldsymbol{t}$ & № & $\boldsymbol{P}_{\text {in }}$ & $\boldsymbol{T}$ \\
\hline 10 & 0.05 & 153 & 24 & 0.08 & 123 & 38 & 0.10 & 91 \\
11 & 0.10 & 88 & 25 & 0.10 & 90 & 39 & 0.11 & 70 \\
12 & 0.11 & 74 & 26 & 0.12 & 60 & 40 & 0.08 & 116 \\
13 & 0.12 & 56 & 27 & 0.11 & 54 & 41 & 0.08 & 109 \\
14 & 0.08 & 114 & 28 & 0.11 & 77 & - & - & - \\
\hline
\end{tabular}

\section{The Main Part of the Paper}

A method had been proposed for determination of remaining resource of the car assemblies (on the example of the most complicated from them (car engine) using:

- density of probability of the basic parameter distribution at the moment of putting the assembly into operation;

- experimental information about operating age of assemblies at the change of the basic parameter from initial occasional value till its ultimate non-occasional value;

- density of probability of the resource distribution from the operating age of this assembly, when the basic parameter reaches the ultimate value..

The technical state of assemblies is changing in monotonous way under the influence of the wear of their rubbing joints. It is possible however to isolate one rubbing joint, which will allow judging in the greatest degree about the ultimate state of the whole assembly by the ultimate state of this junction. $[13,14]$ In this case the assembly demands capital repairs or writing off. The parameter, which describes the change of the mentioned rubbing joint is the basic one, it is substantially correlated with parameters of the rest of rubbing joints. That is why it describes the state of the whole assembly in sufficiently complete way.

As a rule, it is impossible to measure the basic parameter without dismantling the assembly. So, in the current moment of time it is determined in indirect way, by the results of measuring the diagnostic parameter, which is connected functionally with the basic one. In the case to be considered, the consumption of gases, which have been burst into the carter, or the amplitude of vibration of the assembly carter wall on a specific frequency, can serve as such diagnostic parameter.

It is to be noted that it is possible to determine the clearances in the joints of details only before the assemblage, if there are the data about corresponding dimensions of details. In the time of operation the owner of the assembly will do not allow to stop it, dismantle and measure dimensions of details. This will entail the loss of time and loss of part of remaining resource of the assembly with attendant financial expenses for wages for metal craftsmen, purchase of some details (gaskets, locking details) and materials. It is possible to determine the basic parameter for the second time immediately while dismantling the assembly before writing off or during the repair.

Initial data for determination of the assembly remaining resource are following:

- initial value of the basic parameter at the moment of putting the assembly into operation and distribution of density of its probability;
- dependence of operating age of an assembly taking into account conditions of operation from the initial value of the basic parameter on the base of monitoring information;

- value of the basic parameter, which determines the ultimate state of an assembly.

Calculation is carried out in three stages (Figure 1):

- determination of distribution of the basic parameter at the moment of putting the assembly into operation when $t=0$ (lower quadrant of the graph II;

- determination of operating age up to ultimate state of the assembly, which had been put into operation with given value of the basic parameter;

- calculation of remaining resource of the assembly in the form of difference between the value of operating age up to the ultimate state and the current operating age with estimation of the prediction error.

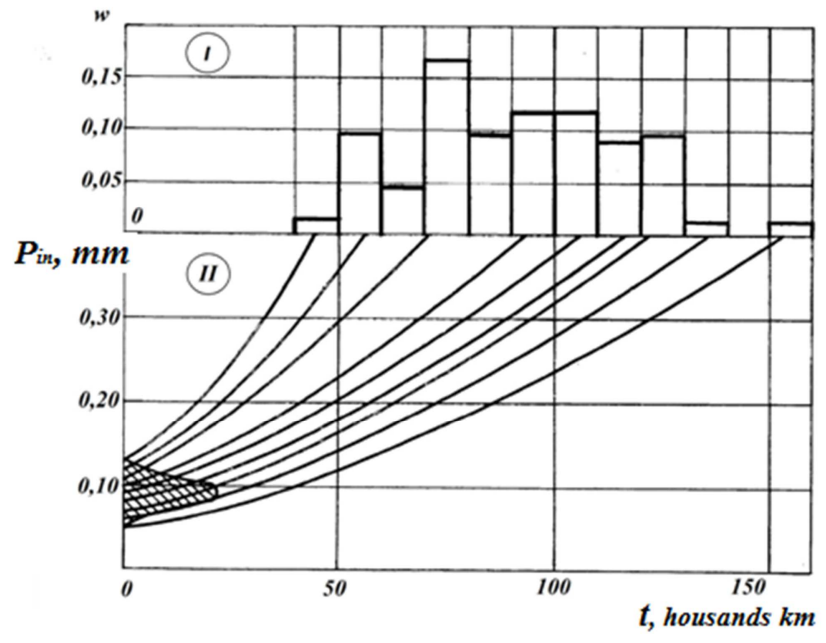

Figure 1. Dependence of operating age tof engines submitted to repair up to the ultimate state from initial clearence $P_{\text {in }}$ between the pistol and cylinder (lower quadrant II) and hystogram of the frequency of refusals $w$ from this operating age (upper quadrant I).

The density of probability for distribution of the basic parameter of an assembly, which had been manufactured or submitted to repair, is distributed (according to information from editions in technology of machine-building and machine repair) by the Hauss curve (this is the hatched field in the figure), as the value of this parameter is formed under the influence of the big number of independent factors (technical level of technological equipment, qualification of workers, values of processing schedules and so on). In this case it is assumed that the expected value of parameter is equal to the half of the tolerance $\delta$ for dimension (of the basic parameter). It is also assumed that six root-mean-square deviations go into the field of tolerance. Then the coefficient of parameter variation is equal to $\delta / 6: \delta / 2 \approx 0.33$. In this case the density of 
probability for distribution of the basic parameter is

$$
f(P)=\frac{6}{\delta \sqrt{2 \pi}} e^{-\frac{\left(0.5 \delta-P_{a v}\right)^{2}}{\delta^{2}}} .
$$

In a number of cases the change of expected value of the basic parameter in dependence on operating age has the nonlinear character (nature), so for increasing the accuracy of prediction it is expedient to use non-linear models, for example, power ones.

The value to be predicted for the operating age of an assembly from the initial value of the basic parameter was expressed by the power equation

$$
t=A P_{\text {in }}{ }^{a} .
$$

Taking logs of the left and right parts of equation (6), we obtain

$$
\lg t=\lg A+\operatorname{alg} P_{\text {in }}
$$

or

$$
t^{\prime}=A^{\prime}+a P_{i n}^{\prime} .
$$

In this expression $t^{\prime}=\lg t ; A^{\prime}=\lg A ; P^{\prime}=P_{i n}$.

With the purpose to find the coefficients of equation (6) by the method of the least squares let us compose the following system of equations

$$
\begin{gathered}
A^{\prime} n+a \sum_{1}^{\mathrm{n}} P_{\mathrm{in}}^{\prime}=\sum_{1}^{\mathrm{n}} t^{\prime}, \\
A^{\prime} \sum_{1}^{\mathrm{n}} P_{\mathrm{in}}^{\prime}+a \sum_{1}^{\mathrm{n}}\left(P_{\mathrm{in}}^{\prime}\right)^{2}=\sum_{1}^{\mathrm{n}} t^{\prime} P_{\mathrm{in}}^{\prime} .
\end{gathered}
$$

here $n$ is the number of observations.

The fit of equation (6) to experimental data is determined by means of the Fisher criterion $F$ with due regard to the number of freedom degrees

$$
F=S_{\mathrm{c}} / S_{\mathrm{r}}
$$

here $S_{\mathrm{c}}$ is the calculated dispersion, $S_{\mathrm{r}}$ - dispersion of reproductivity.

The error of prediction is determined by the confrontation of the numerical and relative values (values to be predicted and experimental ones) of operating age of an assembly up to the ultimate state.

\section{Example}

The value of the basic parameter of engines to be investigated in the form of wear of the group "cylinder - piston" determining the ultimate state of engines $P_{\text {lim }}$ was 0,4 MM. Let us substitute data from the Table 1 into the cited above system of equations (9), then we will obtain

$A^{\prime} 41-a 42.185=80.087$,

$-A^{\prime} 42.185+a 43.710=-82.791$, whence it follows that $A^{\prime}=0.616, A=4.130$ (obtained as a result of taking the antilogarithm of $\left.A^{\prime}\right), a=-1.300$.

Equation (6) in numerical form appears as

$$
t=4.130 P_{\text {in }}^{-1.30}=\frac{4.130}{P_{\text {in }}^{1.30}} .
$$

The expected value of the random quantity $M(t)$ is 94.20 thousands $\mathrm{km}$ of run, calculated dispersion $S_{c}$ is 586.9 , and root-mean-square deviation is $\sigma=24.23$ thousands $\mathrm{km}$ of run. Coefficient of variation $v$ is 0.26 .

The extract of engines from the Table 1 with initial clearance between piston and cylinder equal to $0.10 \mathrm{~mm}$ had been examined with purpose of calculating the dispersion of reproductivity $S_{r}$ for investigated group of engines.. The extract included engines with numbers 3, 9, 11, 20, 25, 35 and 38 . Dispersion of reproductivity was 128.4. The value of the Fisher criterion $F$ was equal to $F=586.9 / 128.4=4.57$. Taking into account the number of degrees of freedom 40 for the greater dispersion and 6 for the lesser one, at the upper limits of deviations of criterion equal to $5 \%$ and $1 \%$, the tabular values of $F_{t}$ are equal to 13.77 and 7.14 , correspondingly, what confirms the satisfactory convergence of experimental and theoretical results.

The distribution of the density of probability $f(t)$ for the resource of assemblies was assumed according to Weibull's law

$$
f(t)=\frac{b}{t_{0}}\left(\frac{t}{t_{0}}\right)^{b-1} e^{-\left(\frac{t}{t_{0}}\right)^{b}}, \text { thousands km, }
$$

in this expression $t_{0}=\left(\bar{t}-C_{a}\right) K_{a}$ and $b$ are parameters of Weibull's distribution; $\bar{t}-$ is the expected value of operating age after repair $t ; K_{a}$ and $C_{a}$ are auxiliary coefficients.

The parameter $t_{0}$ has the dimensional representation of operating age, the parameter $b$ is dimensionless.

The parameter $b$ and auxiliary coefficients $K_{a}$ and $C_{a}$ were determined by the method of interpolation according to the appendix 5 (See [15]). When $v=0.26 b=4.20, K_{a}=0.92$ and $C_{a}=0.23$.

$t_{0}=(94.20-0.23) / 0.92=102.1$, thousands $\mathrm{km}$.

So, taking into account substitution of the found values of parameters, the dependence of the density of probability distribution for after-repair resource can be determined as:

$$
f(t)=\frac{4.2}{102.1}\left(\frac{t}{102.1}\right)^{3.2} e^{-\left(\frac{t}{102.1}\right)^{4.2}}=0.0411(0.0098 t)^{3.2} e^{-0.0098 t^{4.2}}
$$

The experimental distribution of the assembly resource, when the basic parameter reaches the ultimate state $P_{\text {lim }}$ (at the initial value $P_{i n}$ ) is presented in the upper quadrant $I$ of the Figure 1.

Coincidence of data, which have been obtained by the way of equation (13), with experimental information was assured by the way of the Pearson criterion $\chi^{2}$ determined by the equation 


$$
\chi^{2}=\sum_{1}^{n_{y}} \frac{\left(m_{\mathrm{oi}}-m_{\mathrm{Ti}}\right)^{2}}{m_{\mathrm{Ti}}} .
$$

Where $n_{\mathrm{y}}$ is the number of intervals of an enlarged statistic series; $m_{\mathrm{oi}}$ is an experimental frequency in the $i$-th interval of statistic series; $m_{\mathrm{ri}}$ is the theoretical frequency in the $i$-th interval of statistic series.

The value of the Pearson criterion $\chi^{2}$, equal to 4.1 was obtained at the number of degrees of freedom 9. At the probability $90 \%$ the experimental and theoretical information coincide with each other.

The error of prediction was determined by confrontation of numerical (in the form of difference between predicted theoretical $t_{t}$ and the average actual $f_{\text {a.a }}$ operating ages) and relative (in the form of the ratio $\left.\left(t_{a . a}-t_{t}\right) / t_{t}\right)$ values of the assembly operating age up to the ultimate state (Figure 2). The groups of engines, which have been taken into account, were those with the same initial values of the basic parameter $P_{i n}$, including the greatest number of objects to be studied (Table. 3 ).

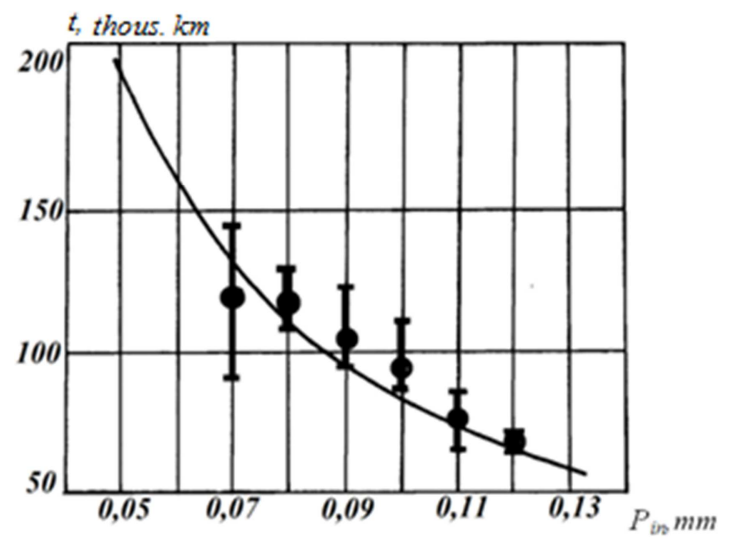

Figure 2. Dependence of predicted operating age t of engines, which were submitted to repair, up to the ultimate state from initial clearance $P_{\text {in }}$ between piston and cylinder, and the average actual values of operating age.

Table 3. Calculation of the error in predicting the operating age of engines up to the ultimate state.

\begin{tabular}{|c|c|c|c|c|}
\hline$P_{i n}, \mathbf{m m}$ & $t_{t}$, thous.km. & $t_{a . a}$, thous. km & $T_{\text {a.a. }}-t_{t}$, thous.km & $\left.\left(t_{a . a}-t_{t}\right) / t_{t}\right)$ \\
\hline 0.07 & 131.0 & 121.0 & -10.0 & -0.083 \\
\hline 0.08 & 110.1 & 118.5 & 8.4 & 0.071 \\
\hline 0.09 & 94.5 & 105.6 & 11.1 & 0.105 \\
\hline 0.10 & 82.4 & 93.2 & 10.8 & 0.116 \\
\hline 0.11 & 72.8 & 71.3 & -1.5 & -0.021 \\
\hline 0.12 & 65.0 & 57.5 & -7 . & -0.130 \\
\hline
\end{tabular}

The prediction of remaining resource of assemblies is especially actual at the operating age coming nearer to the ultimate state. In this case the relative error of prediction changes from 0.021 to 0.130 , what is quite acceptable for using in practice.

\section{Conclusion.}

We introduced and explained the method for determining the operating time of aggregates to their limit state using the monitoring information. Our method makes it possible to calculate the residual life of aggregates in using the difference in operating time to the limit state and the current operating time at the considered time with the forecast error from 2 to $13 \%$. The greatest influence on the operating time of the unit to the limit state has the initial value of the testing parameter.

\section{References}

[1] Fedorov A. G. Forecasting remaining resource of assemblies and units of trucks in the environment of spreadsheet/ A. G. Fedorov, A. M. Krikov, V. N. Delyagin, V. M. Livshits, A. A. Monoszon // Siberian Herald of Agricultural Science. - 2016. - № 3. - P 89-95.

[2] Bazhenov Yu.V. Prediction of the residual resource of constructive elements of vehicles under conditions of operation/ Yu.V. Bazhenov, M.Yu. Bazhenov // Fundamental research. 2015. - № 4. - P. 16-21.

[3] Bazhenov Yu. V. Prediction of the residual life of electronic engine control systems / Yu.V. Bazhenov, V.P.Kalenov // Bulletin of the Siberian State Automobile and Highway Academy. 2017. - № 2 (54). - P. 52-59.

[4] Ismagilov R. N. Prediction of the residual life of the bearing according to the vibration level of the mechanism / R. N. Ismagilov, R. R. Gareev, V. U. Yamaliev, A. A. Matsibor // Exposure Oil Gas. - 2015. - № 3 (42). - P. 65-68.

[5] Desnitsky A. A. Problems of predicting the residual resource of safe operation of fire trucks in the EMERCOM of Russia system / A.A. Desnitsky, N.M. Laurent // Modern technologies for civil defense and emergency response vol. 1. - 2016. - № 1 (7). - P. 110-114.

[6] Kokorev G. D. Methods of selection of diagnosed parameters of cars in the conditions of agricultural production / G. D. Kokorev// Polythematic network electronic scientific journal of the Kuban State Agrarian University. - 2016. - № 117. - P. 793-806.

[7] Da-shuai W. Research on Service Life Prediction of Diesel Engine /X. Xin-peng, W. Feng-zhong, C. Zhi-qiang, Z. DApeng // Journal of Software Engineering, 2016. - № 10. - P. 392-399.

[8] Chebotarev M. I. The expert method of factor analysis of the operational reliability of combine harvesters /M. I. Chebotarev, B.F. Tarasenko, Y. A. Shapiro // Polythematic network electronic scientific journal of the Kuban State Agrarian University. - 2018. - № 136. - P. 71-86.

[9] Richa S. Rana R. Chi-square test and its application in hypothesis testing / S. Richa, R. Rana // Journal of the Practice of Cardiovascular Sciences. - 2015. - № 1 - P. 69-71.

[10] Gurvich, I. B. Operational reliability of automobile engines / I. B. Gurvich, P. E. Syrkin. - M.: Transport, 1984. - 141 p. 
[11] Zhdanovsky, N. S. Reliability and durability of automotive engines / N. S. Zhdanovsky, A. V. Nikolaenko. - M.: Kolos, 1981. $-292 \mathrm{p}$

[12] Mikhlin, V. M. Management of reliability of agricultural machinery / V. M. Mikhlin. - M.: Kolos, 1984.-335 p.

[13] Ivanov, V. P. The influence of the quality of engine repair on their durability / V. P. Ivanov, A. P. Kastryuk // Bulletin of the State Technical University. BY. Sukhoi, 2012. - № 3. - P. 30 34.
[14] Ivanov, V. P. Complex diagnosis of the cylinder-piston group of the internal combustion engine // V. P. Ivanov, A. N. Vaskovich, G. A. Uvarov // Bulletin of the PGU: Industry. Applied Sciences - 2015. - № 11. - P. 87-92.

[15] Reliability and repair of machines / V. V. Kurchatkin [and others]; by ed. V. V. Kurchatina. - M.: Kolos, 2000. - 776 p. 